\title{
Conventional Surgery in Type IV Thoracoabdominal Aortic Aneurysm
}

\author{
Arash Mohammadi Tofigh \\ Imam Hossein Medical Center, Shahid Beheshti University of Medical Sciences
}

Iran

\section{Introduction}

Type IV thoracoabdominal aortic aneurysms (TAAAs) affect the aorta between the diaphragm and aortic bifurcation (Fig. 1). For technical reasons, the aneurysms affecting only the part of aorta from which visceral arteries arise are also categorized as type IV. The level of difficulty of surgical treatment for type IV TAAAs lies between that of type II, which involve descending thoracic and abdominal aorta, and that of the most frequent aneurysm of the aorta: infra renal aortic aneurysm. Like all other TAAAs, the most frequent complication of surgical repair of type IV aneurysms is renal and visceral ischemia. However the risks of pulmonary, spinal and hemorrhagic complications are also important. Except for a few specific cases, surgical repair is carried out in a conventional way according to the technical method explained by Crawford in 1974. Endovascular techniques require specialized teams and will not be addressed in this chapter.

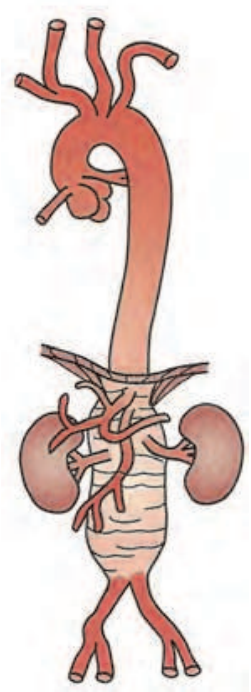

Fig. 1. Type IV thoracoabdominal aortic aneurysm 


\section{Epidemiology and etiology}

Type IV TAAAs represent nearly $20 \%$ of all TAAAs (De Latour et al., 2005). They mostly have atheromatous etiology and develop by a degenerative process identical to infra renal aortic aneurysms. Many of these aneurysms happen in a diseased aorta that has undergone surgery to treat an infrarenal aneurysm, and the degenerative process has reached the upper parts of the aorta. This is mostly seen after an emergency repair of a ruptured infrarenal aortic aneurysm where the technical difficulties of the surgery (or even absence of preoperative imaging) can force the surgeon to suture the prosthesis on a diseased aortic neck. Type IV aneurysms may also develop in previously operated type I and type III aortic dissections and in dissections treated by stent grafts. These types require more complicated treatment than atherosclerotic aneurysms. Rarely, infectious aneurysms, saccular aneurysms secondary to focal weakness in the suprarenal aorta and inflammatory aneurysms associated with Takayasu's disease, are observed.

\section{Indications for surgery}

In contrast to abdominal or thoracic aortic aneurysms, no study has been undertaken on the natural history of type IV TAAAs. This is explained by their relative rarity and the fact that they are generally classified as type II or type III in the literature. Existing data are not sufficient for clinical decision making. Hence, surgeons rely on general data from abdominal aortic aneurysms or other thoracoabdominal types. Except for the symptomatic aneurysms, surgical intervention is usually recommended if the aneurysm diameter reaches $55-60 \mathrm{~mm}$ (Wahlgren \& Wahlberg, 2005). However, several parameters should be considered other than aneurysm diameter: age, general condition, renal and cardiac comorbidities. The ratio of aneurism diameter to the diameter of the normal aorta in each patient must also be considered. For example, a $55 \mathrm{~mm}$ diameter aneurysm developed on an aorta diameter of 20 $\mathrm{mm}$ is considered to be at a higher risk of rupture than one that develops on an aorta diameter of $28 \mathrm{~mm}$ (De Latour et al. 2005). The aortic aneurysm is at risk of rupture if it doubles in diameter compared with the original aorta. The speed of development and the quality of hypertension control should also to be considered, particularly if Marfan's syndrome is present or if the patient has a family history of aortic rupture. Infectious and saccular aneurysms often show a faster and more dangerous pattern of evolution than atherosclerotic aneurysms. Type IV TAAAs are usually seen in subjects over the age of sixty. They impose a clamping of the aorta at the diaphragmatic level which induces visceral and renal ischemia. The surgeon must rely on a clear body of evidence to advise surgery. This reflects the great importance of preoperative assessment in this patient cohort.

\section{Anatomic assessment}

The entire thoracic and abdominal aorta, its visceral and renal branches, iliac arteries and preferably the lower limb arteries, should be evaluated. It is important to study the descending thoracic aorta to verify the presence of ectasia or dual aneurysms that could change the surgical procedure. It also helps in assessing the lower limit of the mural thrombus in the descending thoracic aorta which, if present, might become destabilized by clamping and embolizing the branches. However, small crescents of a few millimeters of mural thrombus located on the descending thoracic aorta are not a contraindication to aortic 
clamping above the celiac trunk. Another important parameter to consider is the distance between the celiac trunk and the neck of the aneurysm, where it will be clamped. Based on the data collected, the surgeon will decide whether to carry out a proximal aortic anastomosis followed by separate reimplantation of the visceral patch, or a single aortic anastomosis encompassing the proximal aorta and the visceral arteries. It is therefore possible, before surgery, to have an idea of the likely duration of clamping of the renal arteries and to predict if protection of the renal parenchyma is needed. The anatomic assessment should include an accurate analysis of visceral and renal arteries (topography and distribution) and the existence of associated stenotic, occlusive or aneurismal lesions that might change the approach or the strategy of surgery. Multi-detector computerized tomography angiography (CTA) can be used to obtain a dynamic analysis of the lesions. It also allows three-dimensional reconstructions and provides all data necessary for the procedure (Fig. 2\&3).

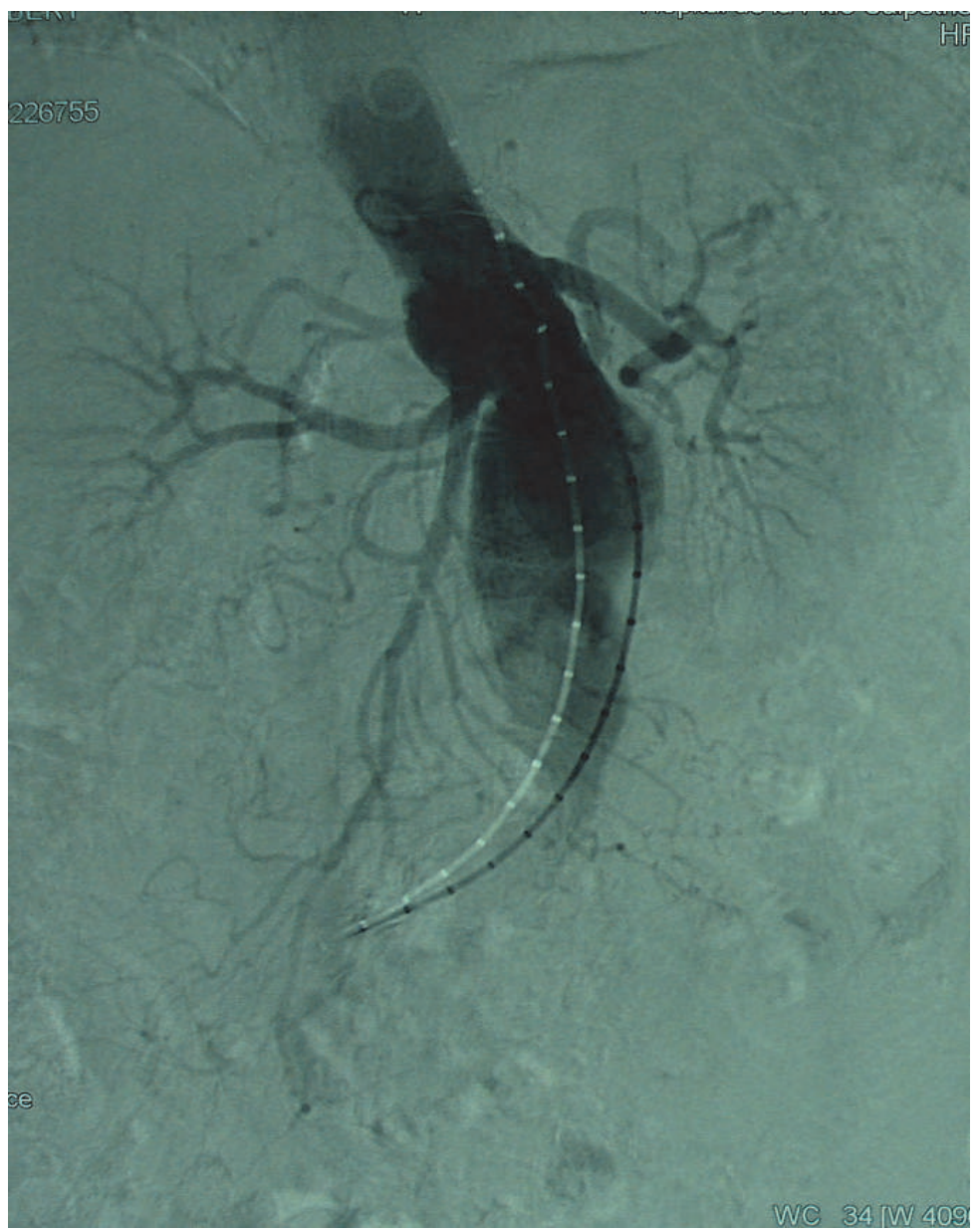

Fig. 2. Angiography of a Type IV thoracoabdominal aortic aneurysm 


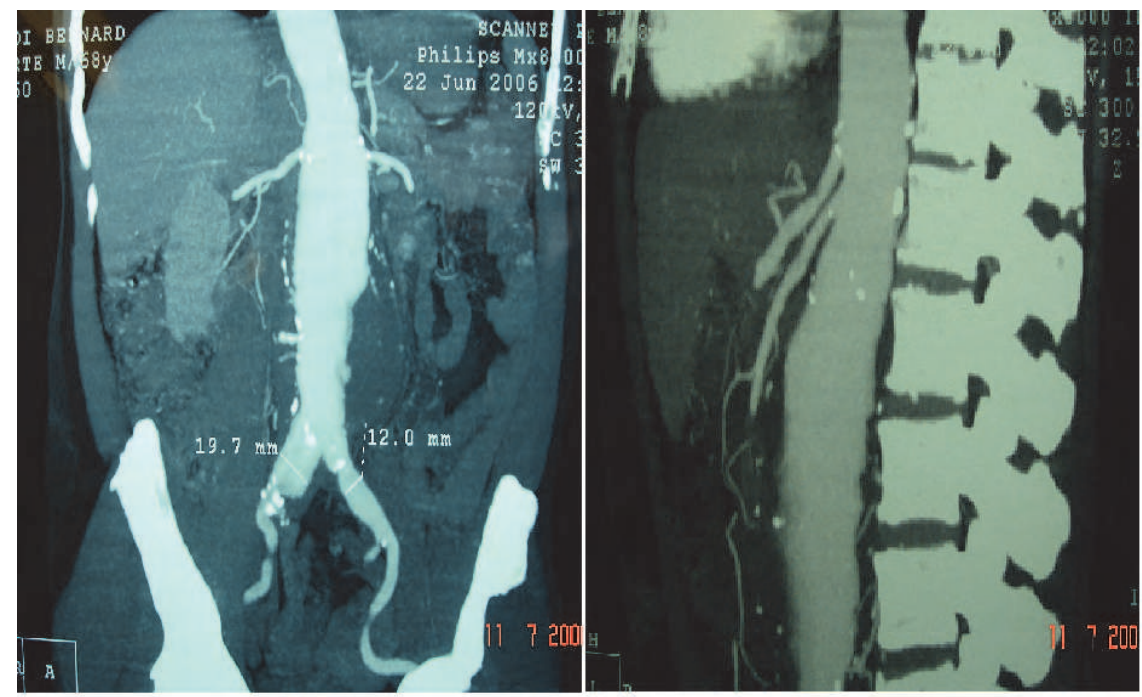

Fig. 3. CT angiography of a type IV thoracoabdominal aneurysm

\section{Preoperative evaluation}

Preoperative evaluation determines the patient's ability to cope with aggressive surgery and allows the team to anticipate possible postoperative decompensation. Four essential systems are checked for these reasons as outlined below.

\subsection{Cardiac}

Surgery for type IV TAAAs requires aortic clamping at the diaphragm level. This causes a sudden increase in cardiac afterload. Conversely, at declamping, there is a sudden collapse in peripheral resistance. In both circumstances, there is a significant increase in cardiac work. Furthermore, many of the patients undergoing this type of surgery are hypertensive and aged $>60$ years. Associated coronary artery disease is also common. The risk of intraoperative acute heart failure is high, so exact preoperative study of myocardial, coronary and valvular status is important (Suzuki et al., 2003).

Undertaking coronary angiography (which is questionable before the surgery of infra renal aortic lesions) is indispensable here, even in the absence of a positive history or clinical signs. An exception may be made in young adults suffering from Marfan's syndrome. Transthoracic echocardiography in search of associated valve diseases is necessary to eliminate severe aortic insufficiency, which would lead to clamping intolerance. An ejection fraction $<30 \%$ or severe coronary lesions should mean temporarily postponement or even canceling the intervention. Transesophageal echocardiography is helpful to evaluate the ascending and descending thoracic aorta, particularly in patients with renal insufficiency. Coronary artery revascularization may be indicated. If coronary stenting is undertaken to achieve this, aneurismal repair should be delayed for 6 weeks, while Clopidogrel should be used. Drug eluting stents might not be used preoperatively. If coronary artery bypass graft (CABG) is selected, use of the left internal mammary artery is not suitable because it is the most important collateral artery to the spinal cord. 


\subsection{Pulmonary}

The surgical approach used for treatment of type IV TAAAs can be a left retroperitoneal or thoracoretroperitoneal approach via a left flank incision, or a thoracoabdominal incision in the tenth left intercostal space. These incisions result in reduction of pulmonary capacity with the possibility of postoperative atelectasis and pleural effusion. Chronic obstructive pulmonary disease (COPD) is common among these patients, and is associated with increased mortality after aneurysm repair. Preoperative assessment of respiratory function can be used to detect patients at risk and help to better prepare them. Pulmonary function tests and arterial blood gas analysis are undertaken routinely. Smoking cessation, appropriate medications and an adequate exercise program improve pulmonary capacity and lower the risk of complications (Knapp et al. 2007).

\subsection{Renal}

Kidneys are frequently associated with postoperative morbidity. Acute renal failure increases mortality and predicts increased non-renal complications. It has been suggested that preoperative renal insufficiency can be a relative contraindication to proceed with repair. Preoperative measurement of blood urea nitrogen (BUN), creatinine clearance, or glomerular filtration is needed. These serve as a benchmark for judging the postoperative functional outcome of the kidneys. This allows explaining to the patient the possibility of temporary or permanent postoperative dialysis if creatinine clearance is $<30 \mathrm{~mL} / \mathrm{min}$. In case of a significant reduction in preoperative creatinine clearance, it is important to have Doppler ultrasound images of the renal arteries and renal parenchyma. A significant functional difference between the kidneys may lead to changes in the surgical strategy to minimize the time of renal ischemia on the best kidney (Biknell et al., 2003). In all cases, detection of renal failure should lead to strengthening the level of renal protection pre- and intraoperatively. Preoperative hydration and avoidance of nephrotoxic agents is also important. Severe renal artery stenosis might be treated pre- or intraoperatively.

\subsection{Carotids}

Assessment of the carotid arteries by Doppler ultrasound is routinely requested to identify a possible cerebral circulatory insufficiency. Even if there is no strong evidence in the literature, it is likely that the hemodynamic changes often encountered during this type of surgery can predispose the patient to stroke. Hence, most surgeons prefer a correction of carotid artery stenosis before considering the repair of type IV aneurysms.

\section{Anesthesia}

During surgery for juxta-diaphragmatic aneurysms, the anesthesiologist will be faced with multiple problems. The right lateral decubitus position, the possible opening of the pleural space and incision of the diaphragm are the most important obstacles for good ventilation. Moreover, COPD is frequently associated with this type of disease. This explains the need for good preoperative chest physiotherapy and the use of bronchodilators. Double lumen endobronchial intubation is not necessary for this type of surgery. During closure of the chest wall, introduction of catheters in the intercostal spaces allows for repeated injections of local analgesics, which is more important just after extubation. The cardiac index should be maintained at $>3$ to ensure good perfusion of tissue. Systolic blood pressure should be 
maintained $~ 100--120 \mathrm{~mm}-\mathrm{Hg}$ to reduce the negative effects of a high afterload on cardiac function. This double constraint (low pressure and high flow condition) is usually provided during the clamping time, through the use of a short acting vasodilator such as sodium nitroprusside. Dobutamine can be added for better cardiac performance. Infusion of nitroprusside should be started a few minutes before clamping of the aorta and stopped minutes before declamping to avoid the risk of cardiovascular collapse due to reperfusion. Blood pressure is then rebalanced by increasing the intravascular volume. A cardiac index $>3$ and a mean arterial pressure $>70 \mathrm{~mm}-\mathrm{Hg}$ are maintained during the first 48 hours after surgery. These measures increase the visceral, renal and spinal perfusion and decrease the prevalence of complication after a low-flow state (Acher et al., 1997).Simultaneous use of different drugs can be difficult, particularly if there is significant bleeding and hypothermia. A short clamping time and cooling of the kidneys cannot guarantee renal protection. To optimize renal perfusion, flow and perfusion pressure are equally important and can be measured intraoperatively. The use of diuretics with osmotic effect (e.g. mannitol) remains controversial. They are used, however, to maintain renal diuresis if postoperative renal failure occurs (De Latour et al., 2005). Bleeding disorders due to clamping of the hepatic circulation can be prevalent. Although minimal doses of heparin are used during surgery, the coagulation profile is often disturbed postoperatively, with a rising prothrombin time and reduced serum fibrinogen level. We can undertake re-transfusion of autologous red packed cells without plasma or clotting factors through cell saver machines. It is therefore important to provide fresh frozen plasma, platelet and fibrinogen just after declamping the aorta. The complementary use of aprotinin provides much more rapid control of postoperative bleeding due to oozing. Oozing is a consequence of hypothermia that is commonly seen in this type of surgery because of the very large incision and opening of the abdominal and thoracic cavities. Hypothermia may promote further arrhythmia that the anesthesiologist must take into account during the procedure (Knapp et al., 2009).

\section{Surgical procedure}

\subsection{Approaches}

Two approaches have been described for surgical treatment of type IV TAAAs. The retroperitoneal approach is by far the most commonly practiced. Left flank incision with resection of the eleventh rib or thoracoabdominal incision in the tenth left intercostal space provide excellent exposure throughout the abdominal aorta, left renal artery and the first centimeters of the superior mesenteric artery (SMA). However, the right renal artery beyond the inferior vena cava, SMA in its distal parts and the hepatic artery remain inaccessible. If these arteries need to be accessed, the transperitoneal approach via a laparatomy is preferred.

\subsubsection{Retroperitoneal approach}

After introduction of a urinary catheter, a central venous catheter, a Swan- Ganz catheter, a radial arterial catheter ( and possibly an intrathecal catheter for drainage of cerebrospinal fluid (CSF)), the patient is positioned in the partial right lateral decubitus with shoulders at $60^{\circ}$ to the operating table and the left hip at $45^{\circ}$. This position allows access to the right femoral artery if necessary. It also facilitates access to the left crus of the diaphragm. The juxta-diaphragmatic aorta can be approached by the thoraco-retroperitoneal route, with resection of the eleventh rib, with or without division of the diaphragm. The two 
approaches are quite similar and differ only by the aortic exposure achieved in the cephalic direction. The incision is made in the left flank in the tenth intercostal space, and the eleventh rib is resected. If the diaphragm is not divided, the exposure of the higher portion of the aorta is less, as well as the diaphragmatic related postoperative complications. Only the left crus of the diaphragm is sectioned and the pleura is often opened in the corner of the incision. However, the gap does not exceed a few centimeters and can be sutured directly at the end of surgery without thoracic drainage. Conversely, if the diaphragm is divided, the aorta can be achieved much higher and control of the descending thoracic aorta is possible. However, the diaphragmatic incision and the significant opening in the pleura which mandate drainage of the thoracic cavity, have negative effects on postoperative pulmonary function.

Two criteria determine the choice between these two surgical approaches, as shown below.

- The exact point where the aneurysm starts and the length of the upper neck of the aneurysm. If the starting point appears on the crus of the diaphragm or slightly below it under CTA, the thoraco-retroperitoneal approach in the tenth intercostal space with resection of the eleventh rib, and isolated dissection of the left crus of the diaphragm without diaphragm incision might be suitable. However, if the aneurismal neck is shorter, the clamp should be placed above the diaphragm and the latter is divided.

- The patient morphotype. If the patient is tall, the costal margin covers broadly the hypochondriac fossa. The diaphragmatic dome is higher, the intercostal spaces are narrower, and exposure of the juxta-diaphragmatic aorta more difficult. Hence, it is better to use a thoraco-retroperitoneal approach in the tenth intercostal space with resection of the eleventh rib and diaphragm incision because it provides better exposure. In short patients with a wide chest, the exposure difficulties are less problematic and the diaphragmatic incision can often be avoided (unless obesity makes it difficult to mobilize the viscera). A flank incision in the tenth intercostal space starting 3- $4 \mathrm{~cm}$ from the spine is made (Fig 4). Its caudal extension is dependent upon the extent of the aneurysm. If there is no involvement of the iliac arteries and reconstruction stops at the aortic bifurcation, the incision goes down near to the umbilicus. If the iliac axes are involved in the aneurysm, the incision stops halfway between the pubic tubercle and the umbilicus. After division of the intercostal muscles and external oblique muscles, the eleventh rib is resected. Blunt dissection of the peritoneum begins when retroperitoneal fat appears and, by mobilizing the peritoneum and its contents medially, the retroperitoneum and aorta are exposed.

This incision allows for the introduction of a self-retaining retractor or a Finochietto retractor between the costal margin and the anterior superior iliac spine. After the retroperitoneal space is entered, the inferior surface of the diaphragm is mobilized medially until the left crus of the diaphragm is fully visualized. The left kidney can be mobilized superiorly and medially to expose the retroperitoneum up to the level of the diaphragm. The ureter should also be identified and mobilized superiorly. The left crus of the diaphragm is divided for exposure of the aorta above the celiac axis. Now the aorta can be readily palpated (especially in its diaphragmatic orifice). If the aorta has a normal size at the orifice, it is not necessary to continue the dissection upward: it will be clamped at this point. If the aneurismal neck is too short or the aneurysm continues cephalically, the diaphragm is divided parallel to its costal attachment. A $2 \mathrm{~cm}$ diaphragmatic fringe is left along the chest wall to ease the final repair. The diaphragmatic incision extends along its attachments to the costal margin, to the left crus that was previously cut. This approach allows finding a safe 
area for clamping the distal descending thoracic aorta. By cutting only the periphery of the diaphragm, injury to the branches of the phrenic nerve is avoided which offers better postoperative pulmonary function. Before clamping and incising the aorta, all para-aortic tissue is transected along the line of the intended aortotomy. Veins lateral to the aorta (e.g. renal lumbar vein) must be tied and transected. The time spent for preparation, reduces bleeding during aortic reconstruction, and helps the anesthesiologist to adjust the hemodynamic.
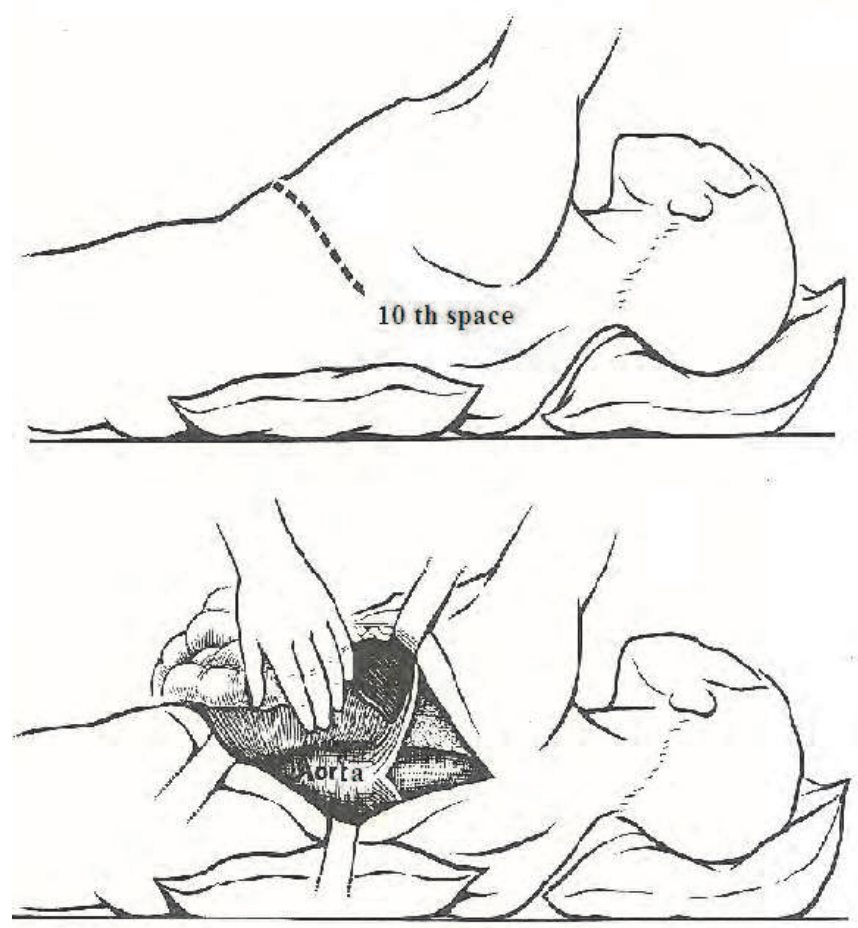

Fig. 4. Flank incision in the $10^{\text {th }}$ intercostals space and exposure of the aorta

\subsubsection{Transperitoneal approach}

The transperitoneal approach via a laparotomy is intended for patients in whom surgical intervention on the right renal artery, common hepatic artery, or distal part of SMA is expected. A midline incision is most frequently used. It should extend from the xiphoid to the symphysis pubis to provide maximal exposure. Once the intra-abdominal contents have been inspected, a left medial visceral rotation is made by releasing the splenic flexure of the colon and mobilizing the spleen and pancreas medially until the suprarenal aorta is exposed. Division of the left crus of the diaphragm provides additional proximal exposure of the suprarenal aorta. The left kidney can be kept in the renal fossa or mobilized medially, which increases the risk of a postoperative pancreatic reaction. By mobilizing the right colon in continuity with the duodenum and shifting the orientation of the SMA and the root of the mesentery, the right renal artery beyond its retrocaval portion is exposed. Finally, the 
common hepatic artery and its branches, and the SMA beyond its original segment are exposed in this approach.

\subsection{Aortic reconstruction}

In most cases of type IV TAAAs, reconstruction of the aorta is by the inclusion technique described by Crawford. Once proximal and distal control is achieved and systemic heparinization initiated, the aneurysm is entered by incising the aorta from its bifurcation distally to the aneurismal neck proximally. Attention should be paid to the ostium of the left renal artery, which is normally situated in the posterior but might be moved upward if the kidney is mobilized superiorly. Hence, the incision should be situated in the left border of the aorta near the spine. If the incision passes near the ostium of the left renal artery, the ostium should be detached with a wide aortic patch (as Carrell patch) that will help reimplantation of the left kidney on the graft. Then the thrombus is evacuated and sent for laboratory analysis. Occlusive catheters are placed in the renal artery, celiac artery and SMA. Backbleeding lumbar arteries, as well as the inferior mesenteric artery are oversewn from within the sac. In this step, the quality of the aortic wall and degree of separation of origins of the renal and visceral arteries should be rapidly assessed. The surgical strategy will be decided based on these data. Two situations are most often encountered as shown below.

1. In most cases, the distance between the celiac trunk and SMA is short. The anastomosis is created at the level of the renal arteries with an oblique technique incorporating the visceral arteries and right renal artery (Fig.5). If the inter-renal distance is not too large, there is no need for separate button reimplantation of the renal arteries. In this situation, the clamping time of the visceral and renal arteries is short (normally $<30$ $\mathrm{min}$ ) with minimal postoperative ischemic complications. The twelfth pair of intercostal arteries is usually included in the patch. However, in most cases, this distance is remarkable and cutting a large patch including the four ostia will leave diseased aortic tissue - the source of secondary dilation. Hence, the left renal artery is treated separately by amputating its origin as a Carrell patch and reimplating it in the graft. (Fig.6)

2. In the case of substantial separation of the origin of the celiac artery and SMA, a convenient technique is to amputate the celiac origin as a Carrell patch, defer its reconstruction, and use a single inclusion button for the SMA and the right renal artery. The left renal artery is treated as described above. The distance between these anastomoses is just a few millimeters and has two drawbacks as shown below.

- If bleeding occurs from the suture line after declamping, repair would be difficult. The proximity of the two suture points between the prosthesis and the aorta does not allow mobilization and exposure of the posterior suture line. To reduce this risk, it seems preferable to completely sever the aorta circumferentially and to not carry out the in-lay suture technique. This allows for better visualization of the passage of sutures and safer anastomosis.

- Commonly there is a pair of large intercostal arteries between the celiac trunk and the upper limit of the aneurysm. The narrow spaces in this area do not allow for another anastomosis for the intercostal arteries, hence, they should be included in one of the visceral patches. Ligation or thrombosis of the intercostal arteries is probably one of the causes of postoperative paraplegia encountered in this type of surgery.

Once the aorto-prosthetic anastomosis is realized, the celiac axis, SMA and right renal artery are reimplanted as an inclusion button in the graft. Then the left renal artery is reimplanted 
or bypassed separately in the tube graft. (Fig.7) This anastomosis should be placed on the left postero-lateral side of the graft to avoid kinking of the artery after repositioning the left kidney. The inferior mesenteric artery is in general ligated and finally the distal anastomosis done. This anastomosis can be created between a tube graft and the distal portion of the aorta, or between a bifurcated graft and both iliac arteries (including hypogastric arteries) depending on the extent of the aneurysm. Colonic perfusion can be examined by opening the peritoneum and, if sigmoidal ischemia is present, the inferior mesenteric artery should be reimplanted. Protamine is then administered and extensive hemostasis undertaken. The aneurismal layer is closed over the graft, and the muscular layers of the crus firmly closed. The diaphragm is repaired with heavy absorbable sutures and attached to the parietal flap, left in situ during the initial section. A chest tube is inserted and the retro peritoneum drained. Two catheters can be positioned on either side of the tenth intercostal space to infuse analgesic in the postoperative phase.

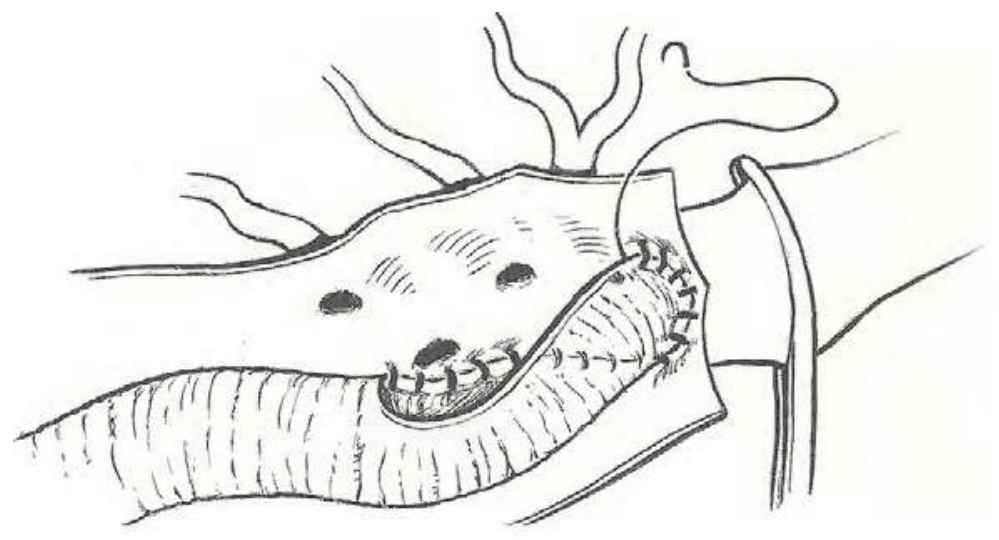

Fig. 5. Anastomosis between the aorta and graft including visceral and renal arteries

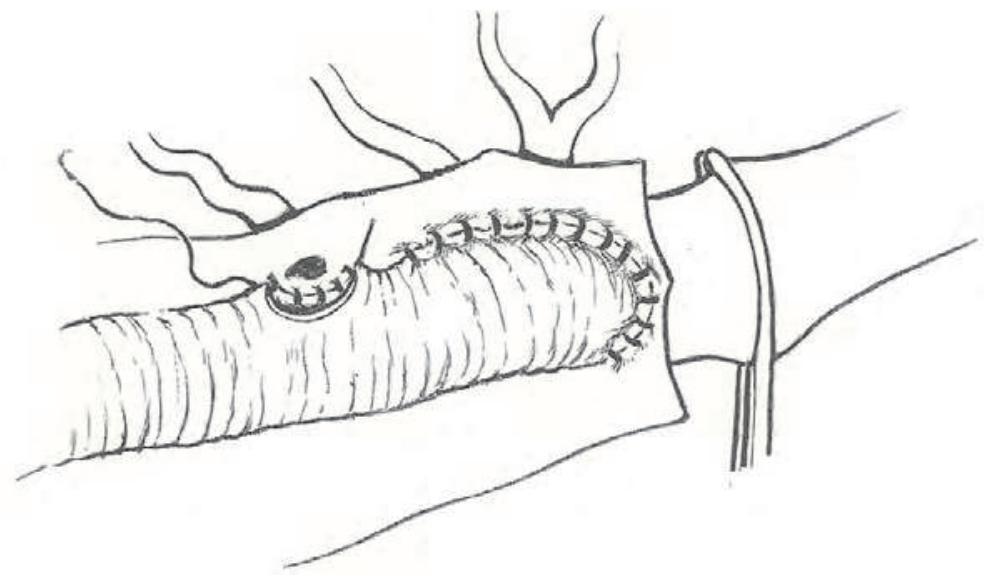

Fig. 6. Separate anastomosis of the left renal artery in the graft 

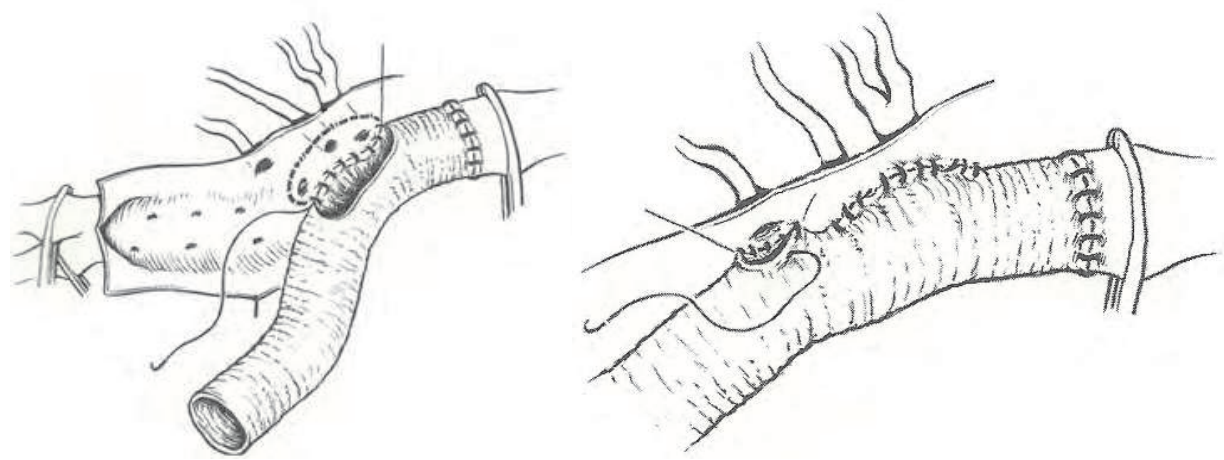

Fig. 7. Separate anastomosis between the graft and the aorta and the visceral patch

\subsection{Spinal protection}

As mentioned above maintaining good hemodynamic is the main protective method for perfusion of the spinal cord. The other protective method for the spinal cord in this type of surgery is to include the ostia of the lowermost pair of intercostal arteries in the aortic anastomosis or in the visceral patch. If the upper anastomotic line lies above the diaphragm, cerebrospinal fluid (CSF) drainage is carried out (Safi et al., 2003). There is no need for extracorporeal circulatory support.

\subsection{Renal protection}

The prevalence of postoperative renal failure varies between $0 \%$ and $21 \%$ (table 1 ). It is mainly dependent upon the initial renal function, clamping time, and quality of the surgical procedure (postoperative occlusion of a renal or polar artery). In our surgical unit, the best results are obtained if preoperative renal function is in the normal range, the upper anastomotic patch includes both renal arteries and the duration of renal clamping is $<30$ min. Under these conditions intraoperative renal protection is not needed. If the patient has preoperative renal failure, then each kidney is perfused with $300 \mathrm{~mL}$ of cold $\left(+4{ }^{\circ} \mathrm{C}\right)$ Ringer's lactate solution after opening the aneurysm. The infusion is undertaken for $4-5$ min at $150 \mathrm{~mm}-\mathrm{Hg}$ through a coronary cannula. Such high pressure is important to eliminate micro-thromboses in the capillary bed (as has been demonstrated in kidney transplantation). Proximal anastomosis is then carried out. If the repair is more complex, with separate reimplantation of both renal arteries, the clamping time might be much longer. It is preferable to carry out renal cold perfusion with the same technique but it is repeated for the left renal artery, after declamping the right renal artery. Finally, maintaining a high cardiac output and mean arterial pressure $>70 \mathrm{~mm}-\mathrm{Hg}$ during and after surgery are two major elements to ensure satisfactory diuresis. This maintenance of good hemodynamic is also necessary for protecting the spinal cord (Coselli, 2010).

\section{Special conditions}

\subsection{Dissecting aneurysms}

A descending thoracic aortic dissection can be transformed to a type IV TAAA several months or even years after the acute episode (Conrad et al., 2010). The external diameter of 
the descending thoracic aorta may be conserved near the normal range as it expands slightly, whereas immediately after crossing the diaphragm a significant expansion occurs, (especially if an intimal tear is present between the diaphragm and renal arteries). Technically the aortic wall is thick and sclerotic and does not pose a problem for suturing. There are, however, two other practical challenges:

- The proximal anastomosis will be carried out on a dissected area. Hence, the inner membrane should be resected to create a wider stoma. The suture should be placed on the outer layer. If a parietal thrombus is present, it should be evacuated to avoid embolization upon declamping.

- The existence of a false channel strongly modifies the endoaortic appearance. Unlike atherosclerotic aneurysms, ostia of the renal and visceral arteries are not always easily distinguished (especially if the false lumen ends on an ostium or it continues along the branched artery). Simple resection of the inner membrane leaves a fibrous bead in the origin of the artery which can induce postoperative thrombosis. This bead should not be included in the prosthetic suture line. It is better to bury it in the aortic wall with a few sutures to eliminate local turbulence. If the false lumen extends to the right renal artery it is recommended to detach it from the aorta. It will be later reimplanted on the aortic graft with a short prosthetic graft.

\subsection{Simultaneous occlusive lesions}

Preoperative evaluation may reveal significant stenosis of renal and visceral arteries. If located on the left renal artery (or if purely ostial), they can be treated by the Crawford technique described above. However, in cases of extensive lesions on the right renal artery, SMA or hepatic artery, this technique is no longer possible due to the incision not exposing the distal parts of the arteries. There are several options to solve this condition:

- Balloon expandable angioplasty of the diseased arteries before surgery can be a convenient technical expedient.

- Using a laparotomy approach in which the spleen and pancreas are mobilized medially until the suprarenal aorta is exposed (as described in transperitoneal approach section). This approach allows extensive dissection of diseased arteries and carrying out reconstruction by a bypass from the prosthesis.

- If a long segment of the SMA is involved or if a prolonged ischemia on the left dominant kidney should be avoided, revascularization can be achieved first on the visceral arteries. The left diaphragm and its crus are completely divided and the distal $5-6 \mathrm{~cm}$ of the thoracic aorta exposed. The aorta is then partially clamped by a side biting clamp and a graft anastomosed at this level to the aorta. It is then used for antegrade revascularization of the visceral or renal arteries. Once visceral revascularization has been completed, the aorta is clamped and the procedure continues as described above (Ballard et al., 2002).

- It is possible to carry out this surgery in two stages. Extra-anatomic revascularization of occluded arteries (hepato-renal or right ilio-mesenteric bypass) or kidney autotransplantation can be done in the first surgery. The patient is then operated a few days or weeks later for treatment of the aneurysm. The disadvantage of this approach is the multiplication of surgical approaches and surgical risks. However, in some cases, this two-step approach, can minimize the risk of visceral or renal ischemia during the clamping of the aorta, and thus should be considered (Etz et al., 2010). 


\subsection{Retro-aortic left renal veins}

As an anatomic variation the left renal vein can be retro-aortic. In this case, by mobilizing the kidney anteriorly during retroperitoneal exposure, the renal vein completely ties around the aneurysm and hinders incision and opening of the aorta. Hence, there is no choice apart from renal auto-transplantation. The left kidney should be completely released and its ureter dissected down to the iliac vessels. The renal artery is cut near its ostium and the renal vein cut close to the spine. The kidney is then washed and cooled continuously in a sterile tray positioned above the pubis while the ureter is still contiguous. Once the aortic reconstruction is completed, the kidney is auto-transplanted to the iliac vessels.

\section{$\mathbf{8 . 4}$ Isolated aneurysm of the visceral part of the aorta}

Aneurysms affecting only the visceral part of the aorta where the SMA and celiac arteries branch, are mostly saccular with infectious etiology (Fig.8) or are secondary to rupture of a calcified plaque. In these cases, it is recommended to excise the aneurismal sac and to repair the aortic wall with a Dacron patch or cryopreserved arterial homograft. The latter material is preferred if infectious or mycotic etiologies are suspected (Vogt et al., 1998). This repair is not always readily undertaken due to the poor quality of the aortic wall and a resection that is wider than expected. Also, the ostium of the aneurysm may be near the origin of the renal or visceral arteries, which makes simple repair unachievable. In these cases, it is recommended to do the reconstruction by the Crawford's inclusion technique described above.

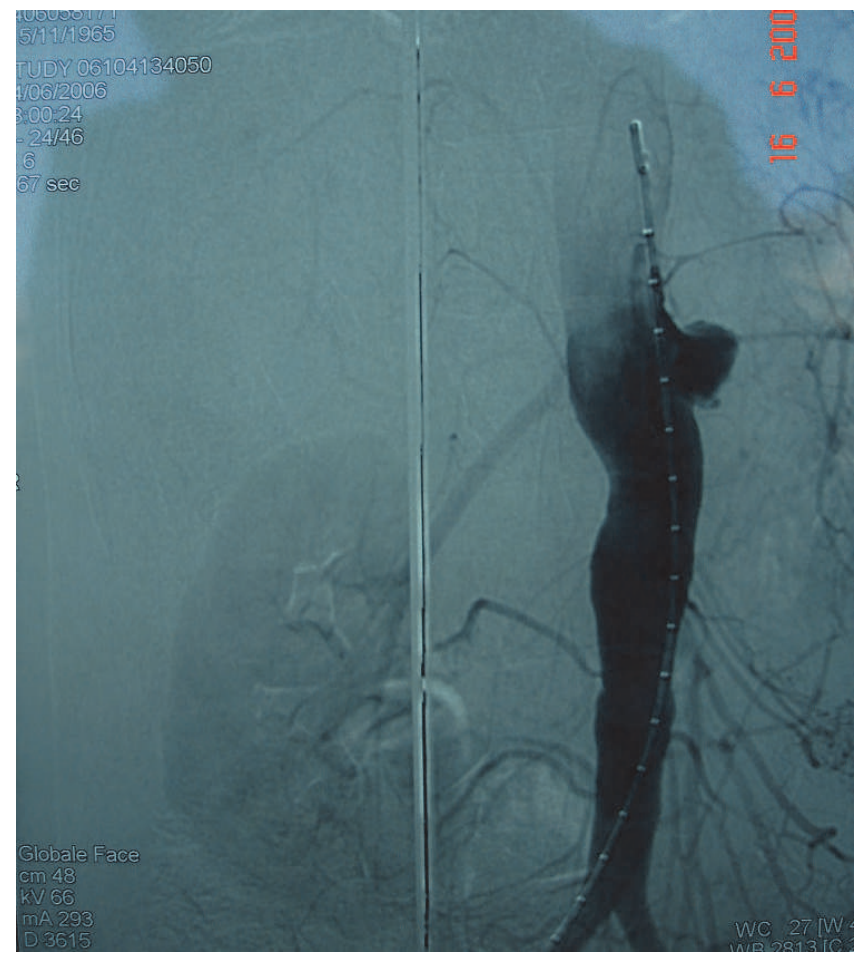

Fig. 8. A saccular aneurysm of the suprarenal part of the aorta with infectious origin 


\subsection{Reoperations}

Surgical treatment of type IV TAAAs (like any prosthetic reconstruction) is subject to possible secondary degradation (Dardick et al., 2001). The occurrence of an anastomotic pseudoaneurysm or dilatation of the aortic patch may require further surgery if the lesion diameter becomes unacceptably large. Reoperation and a direct approach to the lesion are not easy and, since the advent of aortic stent grafts, a hybrid technique is preferred (Patel et al., 2009). In this technique, retrograde revascularization of the visceral arteries is followed by covering the aneurysm by a stent graft.

After laparotomy, visceral arteries are dissected a few centimeters from the aorta and an 8mm Dacron graft is anastomosed to a common iliac artery or to the infrarenal aorta. The SMA is then ligated at its origin and an end to side anastomosis made between it and the right side of the Dacron graft. The graft is then carefully tunneled beneath the pancreas and an end to side anastomosis made between it and the common hepatic artery. The celiac trunk is then ligated at its origin. A bifurcated Dacron graft is then anastomosed on the same iliac artery, the contralateral iliac artery or on the infrarenal aorta to revascularize the renal arteries. Once renal and visceral revascularization is confirmed, an aortic stent graft is placed to cover the aneurysm (Fig.9).

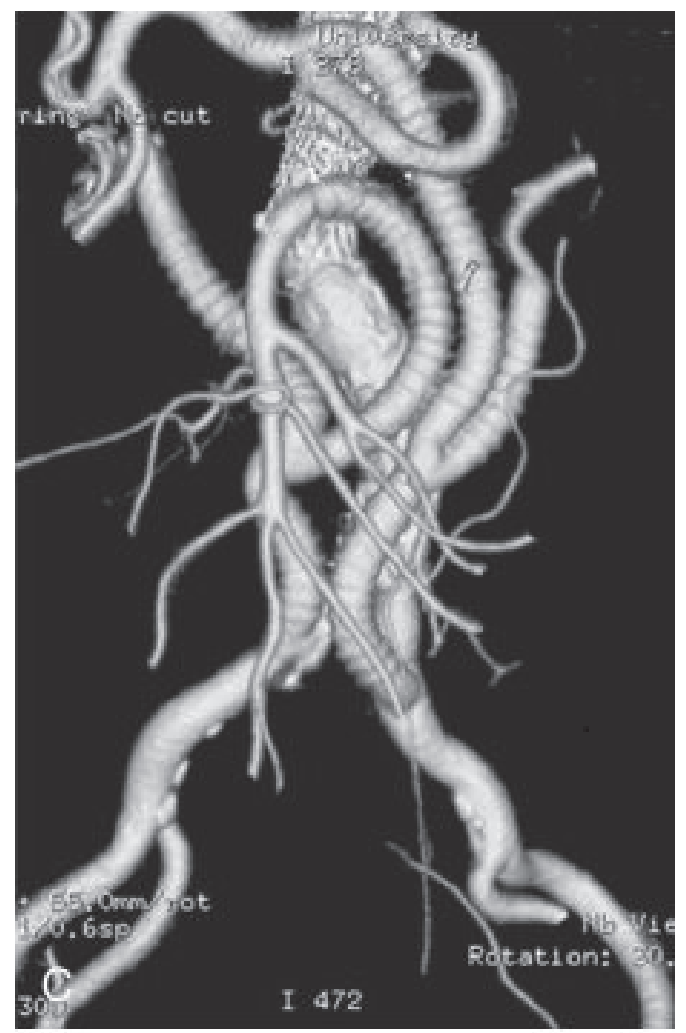

Fig. 9. Postoperative CTA of a hybrid reconstruction of a type IV thoracoabdominal aneurysm 


\section{Results}

Results of the studies conducted on type IV TAAAs which are published in the literature are summarized in Table 1. Some results correspond to series with only type IV aneurysms; others have been extracted from series covering all TAAAs. The overall postoperative mortality is higher than that for infrarenal aneurysms, and is 3.6\% -- $17 \%$ ( mean 8.8\%). Most deaths are due to multi-organ failure secondary to low-cardiac output and renal failure. Postoperative bleeding is also a significant cause of death, immediately after multi-organ failure. The differences found among these studies might be caused by "The center effect". The prevalence of postoperative renal failure is difficult to analyze because the criteria are highly variable from one series to another. The risk of postoperative dialysis is much more dependent upon preoperative renal function than on clamping time. Overall, in the contemporary series, the prevalence of dialysis after surgery varies from $0 \%$ to $21 \%$ ( mean $9 \%$ ). The occurrence of postoperative renal failure requiring dialysis significantly increases postoperative mortality. In surgery for type IV TAAAs, spinal cord ischemia due to aortic clamping is less common than for surgery for type II or III. The prevalence of paraplegia varies from $0 \%$ to $4 \%$ in elective surgery and from $0 \%$ to $20 \%$ in urgent and emergency surgery. There is no evidence supporting that further protective measures (CSF drainage, extracorporeal circulatory support, visceral perfusion) reduce the risk of this complication. When the anastomoses of the upper neck of the aorta and the visceral patch are separated, CSF drainage appears to be the logical approach. Also, in these patients clipping or ligating the lower intercostal arteries which may arise between the two anastomoses should be avoided (Safi et al., 2003).

\begin{tabular}{|c|c|c|c|c|c|}
\hline Author & $\begin{array}{c}\text { Period of } \\
\text { study }\end{array}$ & $\begin{array}{c}\mathbf{n} \\
\text { (patients) }\end{array}$ & $\begin{array}{c}\text { Paraplegia } \\
\mathbf{n}(\%)\end{array}$ & $\begin{array}{c}\text { Renal insufficiency } \\
\mathbf{n}(\%)\end{array}$ & $\begin{array}{c}\text { Mortality } \\
\mathbf{n}(\%)\end{array}$ \\
\hline Richards et al. & $2000-2010$ & 53 & $1(2)$ & - & $3(6)$ \\
\hline Ockert et al. & $1997-2004$ & 30 & $1(1.6)$ & $1(1.6)$ & $2(6)$ \\
\hline Lombardi et al & $1993-2003$ & 56 & $0(0)$ & $2(4)$ & $7(13)$ \\
\hline Schepens et al. & $1981-2003$ & 42 & $2(5)$ & $4(6)$ & $3(7)$ \\
\hline Coselli et al. & $1986-2001$ & 329 & $6(2)$ & $22(7)$ & $12(4)$ \\
\hline Cina et al. & $1990-2001$ & 42 & $0(0)$ & $9(21)$ & $2(5)$ \\
\hline Cambria et al & $1987-2001$ & 66 & $1(1.5)$ & - & $5(8)$ \\
\hline Bicknell et al. & $1993-2001$ & 130 & $6(5)$ & $20(15)$ & $22(17)$ \\
\hline Ballard et al. & $1996-2001$ & 20 & $2(6)$ & $0(0)$ & $2(6)$ \\
\hline
\end{tabular}

Table 1. Results of conventional surgery of type IV thoracoabdominal aortic aneurysm. 


\section{Conclusion}

Open repair of type IV TAAA may be undertaken using different approaches with acceptable levels of morbidity and mortality. The surgery is not as difficult as that for type II or III TAAAs. Compared with the surgical procedure described by Crawford for TAAAs, (which requires wide opening of the thorax and abdomen, spinal cord protection, reimplantation of the intercostal arteries and usually extracorporeal circulatory support) this intervention is simpler and could be described as "modified Crawford procedure". Despite this relative simplicity, surgical risks should not be underestimated. A specialized team is required in all cases, especially for the management of hemodynamic complications.

\section{Acknowledgments}

I would like to thank Dr. K. Sheibani for his help in preparing this chapter and the staff of the Clinical Research Center of Imam Hossein Medical Center for their support.

\section{References}

Acher , CW; Wynn, MM; Hoch, JR \& Kranner, PW.(1998). Cardiac function is a risk factor for paralysis in thoracoabdominal aortic replacement. Journal of Vascular Surgery, Vol. 27, No. 5,( May 1998), pp. 821-828

Ballard, JL; Abou Zam-Zam, AM \& Teruya,TH. (2002). Type III and IV thoracoabdominal aortic aneurysm repair: result of a trifurcated two graft technique. Journal of Vascular Surgery, Vol. 3, No. 2, (August 2002), pp. 211-216

Biknell, CD; Cowan, AR \& Kerle MI. (2003). Renal dysfunction and prolonged visceral ischemia increase mortality rate after suprarenal aneurysm repair. British Journal of Surgery, Vol. 90, No. 9, (September 2003), pp. 1142-1146

Cambria, RP; Clouse, WD; Davidson, JK; Dunn, PF \& Corey, M.(2002). Thoracoabdominal aneurysm repair: results with 337 operations performed over a 15 year interval. Annals of Surgery, Vol.236, No.4, (October 2002), pp. 471- 479

Cina, CS; Lagana, A \& Bruin, G. (2002). Traitement chirurgical des anévrysmes de l'aorte thoraco-abdominale : étude prospective d'une cohorte de 121 malades. Annales de Chirurgie Vasculaire, Vol. 16, (2002), pp. 631-638

Coselli, JS. (2010). Strategies for renal and visceral protection in thoracoabdominal aortic surgery. Journal of Thoracic and Cardiovascular Surgery, Vol. 140, No. 6 Suppl, ( December 2010), pp. S147-149

Coselli, JS; Conklin, LD\& Lemair, SA. (2002). Thoracoabdominal aortic aneurysm repair: review and update of current strategy. Annals of thoracic surgery, Vol. 74, No. 5, (November 2002), pp. S1881-S1884

Conrad, MF; Chung, TK; Cambria, MR; Paruchuri, V; Brady, TJ \& Cambria RP. (2010). Effect of chronic dissection on early and late outcomes after descending thoracic and thoracoabdominal aneurysm repair. Journal of Vascular Surgery, Vol. 53, No. 3, (March 2010), pp. 600-607

Crawford,ES. (1974). Thoraco-abdominal and abdominal aortic aneurysms involving renal, superior mesenteric and coeliac arteries. Annals of Surgery, Vol. 179, No. 5, (May 1974), pp. 763-772 
Dardick, A; Perler,BA; Rose Borough, GS \&Williams, GA.(2001) Aneurismal expansion of The visceral patch after thoracoabdominal aortic replacement, an argument for limiting patch size. Journal of Vascular Surgery, Vol. 36, No.3, (September 2001), pp.405-409

De Latour, B; Camilleri, JP ; Nourissat, G; Favre, JP \& Barral, X. (2005). Traitement chirurgical conventionnel des anévrysmes thoraco-abdominaux de type IV, In: Traitement des anévrysmes de l'aorte abdominale, Kieffer \& Koskas, pp. 83-95, Editions AERCV, ISBN: 2-907232-19-3, Paris

Etz ,CD; Zoli, S; Mueller, CS; Bodian, CA; Di Luozzo, G; Lazala, R; Plestis, KA \& Griepp, RB. (2010). Staged repair significantly reduces paraplegia rate after extensive thoracoabdominal aortic aneurysm repair Journal of thoracic and cardiovascular surgery, Vol.139, No. 6, (June 2010), pp. 1464-1472

Knapp, J; Bernhard, M; Rauch, H; Hyhlik-Dürr, A; Böckler, D \& Walther A.(2009). Anesthesiologic procedures for elective aortic surgery, Anaesthesist, Vol. 58, No. 11, (November 2009), pp. 1161-1182

Lombardi, JV; Carpenter, JP; Pochetino,A; Sonnad, SS \& Bavaria, JE. (2003). Thoracoabdominal aortic aneurysm surgery repair after prior aortic surgery. Journal of Vascular Surgery, Vol. 38, No.6, (December 2003), pp. 11851190

Ockert, S; Riemensperger, M; Von Tengg-Kobligk, H; Schumacher, H; Eckstein, HH \& Böckler, D.(2009). Complex abdominal aortic pathologies: operative and midterm results after pararenal aortic aneurysm and typeIV thoracoabdominal aneurysm repair. Vascular, Vol.17, No.3, (May- June 2009), pp. 121-128

Patel, R; Conrad, MF; Paruchuri, V; Kwolek, CJ; Chung, TK \& Cambria, RP.(2009). Thoracoabdominal aneurysm repair: hybrid versus open repair. Journal of Vascular Surgery, Vol. 50, No. 1, (July 2009), pp. 15-22

Richards, JM; Nimmo, AF; Moores, CR; Hansen, PA; Murie, JA \& Chalmers, RT. (2010). Contemporary results for open repair of suprarenal and type IV thoracoabdominal aortic aneurysms. British Journal of Surgery, Vol. 97, No. 1, (January 2010), pp. 45-49

Safi, Hj; Miller, CC $3^{\text {rd }} \&$ Huynh, TT. (2003). Distal aortic perfusion and cerebrospinal fluid drainage for thoraco abdominal and descending thoracic aortic repair: ten years of organ protection. Annals of surgery, Vol. 238, No.3, (September 2003), pp. 372-381

Schepens, M; Dossche, K \& Morshuis, W.(2004). Introduction of adjuncts and their influence on changing results in 402 consecutive thoracoabdominal aortic aneurysm repair. European Journal of Cardiothoracic Surgery, Vol. 25, No. 5, (May 2004), pp. 701-707

Suzuki, S; Davis 3rd, CA; Miller 3rd, CC; Huynh, TT; Estrera, AL; Porat, EE; Vinnerkvist, A \& Safi, HJ. (2003). Cardiac function predicts mortality following thoracoabdominal and descending thoracic aortic aneurysm repair. European Journal of Cardiothoracic Surgery, Vol. 24, No. 1, (July 2003), pp. 119-124 
Vogt, PR; Brunner-La Rocca, HP; Carrel, T; von Segesser, LK; Ruef, C; Debatin, J; Seifert, B; Kiowski, W \& Turina ,MI.(1998). Cryopreserved arterial allografts in the treatment of major vascular infection: a comparison with conventional surgical techniques. Journal of Thoracic and Cardiovascular Surgery, Vol. 116, No. 6, (December 1998), pp. 965-972

Wahlgren, CM \& Wahlberg, A. (2005). Prise en charge des anévrysmes thoraco-abdominaux de type IV, In: EMC Techniques chirurgicales, chirurgie vasculaire, pp. 43-152, Elsevier Sas, Paris 


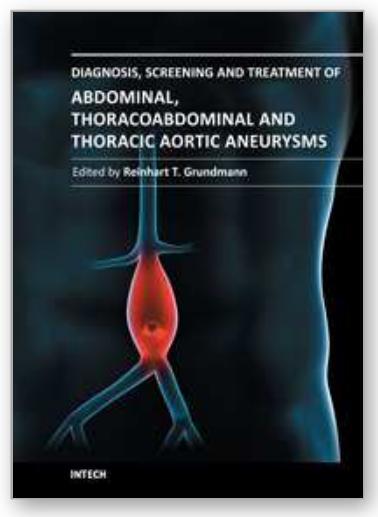

\author{
Diagnosis, Screening and Treatment of Abdominal, \\ Thoracoabdominal and Thoracic Aortic Aneurysms \\ Edited by Prof. Reinhart Grundmann
}

ISBN 978-953-307-466-5

Hard cover, 414 pages

Publisher InTech

Published online 12, September, 2011

Published in print edition September, 2011

This book considers mainly diagnosis, screening, surveillance and treatment of abdominal, thoracoabdominal and thoracic aortic aneurysms. It addresses vascular and cardiothoracic surgeons and interventional radiologists, but also anyone engaged in vascular medicine. The high mortality of ruptured aneurysms certainly favors the recommendation of prophylactic repair of asymptomatic aortic aneurysms (AA) and therewith a generous screening. However, the comorbidities of these patients and their age have to be kept in mind if the efficacy and cost effectiveness of screening and prophylactic surgery should not be overestimated. The treatment recommendations which will be outlined here, have to regard on the one hand the natural course of the disease, the risk of rupture, and the life expectancy of the patient, and on the other hand the morbidity and mortality of the prophylactic surgical intervention. The book describes perioperative mortality after endovascular and open repair of AA, long-term outcome after repair, and the cost-effectiveness of treatment.

\title{
How to reference
}

In order to correctly reference this scholarly work, feel free to copy and paste the following:

Arash Mohammadi Tofigh (2011). Conventional Surgery in Type IV Thoracoabdominal Aortic Aneurysm, Diagnosis, Screening and Treatment of Abdominal, Thoracoabdominal and Thoracic Aortic Aneurysms, Prof. Reinhart Grundmann (Ed.), ISBN: 978-953-307-466-5, InTech, Available from:

http://www.intechopen.com/books/diagnosis-screening-and-treatment-of-abdominal-thoracoabdominal-andthoracic-aortic-aneurysms/conventional-surgery-in-type-iv-thoracoabdominal-aortic-aneurysm

\section{INTECH}

open science | open minds

\section{InTech Europe}

University Campus STeP Ri

Slavka Krautzeka 83/A

51000 Rijeka, Croatia

Phone: +385 (51) 770447

Fax: +385 (51) 686166

www.intechopen.com

\section{InTech China}

Unit 405, Office Block, Hotel Equatorial Shanghai

No.65, Yan An Road (West), Shanghai, 200040, China

中国上海市延安西路65号上海国际贵都大饭店办公楼405单元

Phone: $+86-21-62489820$

Fax: $+86-21-62489821$ 
(C) 2011 The Author(s). Licensee IntechOpen. This chapter is distributed under the terms of the Creative Commons Attribution-NonCommercialShareAlike-3.0 License, which permits use, distribution and reproduction for non-commercial purposes, provided the original is properly cited and derivative works building on this content are distributed under the same license. 